\title{
Accuracy of Dobutamine Stress Echocardiography in Detecting Coronary Artery Disease
}

\author{
Pamela A. Marcovitz, MD, and William F. Armstrong, MD
}

\begin{abstract}
The diagnostic accuracy of dobutamine stress echocardiography (DSE) (incremental infused doses of 5, 10, 20 and $30 \mu \mathrm{g} / \mathrm{kg} / \mathrm{min}$ ) was evaluated in 141 patients who underwent coronary arteriography within 2 weeks of DSE. All patients were being evaluated for known or suspected coronary artery disease (CAD). DSE was interpreted blindly as normal or showing evidence of CAD, depending on the presence of resting or inducible wall motion abnormalities. Coronary arteriograms were reviewed in a blinded, quantitative fashion. DSE had a sensitivity of $96 \%$ for detecting patients with CAD, and a specificity of $66 \%$. For the 53 patients with normal resting wall motion, sensitivity was $87 \%$ and specificity $91 \%$. The protocol was well-tolerated by all patients. In comparison with wall motion analysis, 12-lead electrocardiograms during dobutamine infusion revealed ischemic changes in only $17 \%$ of patients with CAD. It is concluded that DSE is a clinically useful and accurate means for detecting CAD, its specificity is hindered in patients with resting wall moton abnormalitles, and it can safely be used in pathents with known cardiac disease.
\end{abstract}

(Am J Cardiol 1992,69:1269-1273)

From the Division of Cardiology, Department of Internal Medicine, School of Medicine, University of Michigan, Ann Arbor, Michigan. Manuscript received December 31, 1991; revised manuscript received and accepted January 21, 1992.

Address for reprints: William F. Armstrong, MD, Echocardiography Laboratory, University Hospital, UHB1F215, 1500 East Medical Center Drive, Ann Arbor, Michigan 48109-0022. $r$ ardiovascular stress testing is a frequently used technique for diagnosing occult coronary artery disease (CAD), determining prognosis in patients after myocardial infarction and in risk stratification in patients with documented CAD. The limitations of electrocardiographic and symptom analysis during treadmill testing have been well-documented, and imaging with thallium scintigraphy or radionuclide ventriculography has been shown to improve the sensitivity and specificity of treadmill testing. ${ }^{1}$ More recently, in established, experienced echocardiographic laboratories, 2-dimensional echocardiographic imaging was performed during stress testing, yielding an increased sensitivity and specificity equivalent to that seen with more established nuclear medicine techniques. ${ }^{2-4}$ For patients unable to exercise, alternate strategies for cardiovascular stress testing are necessary. The most frequently used alternative strategy is vasodilator stress testing with either dipyridamole or adenosine, in conjunction with thallium perfusion imaging. ${ }^{5}$ More recently, interest was shown in using combined chronotropic and inotropic stimulation with dobutamine as a means of cardiovascular stress testing in patients unable to exercise. ${ }^{6-9}$ This study prospectively evaluates the clinical use and diagnostic accuracy of dobutamine stress echocardiography (DSE) in patients with known or suspected CAD.

\section{METHODS}

Patient population: Between January 1990 and May 1991, 568 patients underwent DSE at the University of Michigan. Cardiac catheterization with coronary arteriography was performed in 141 of these patients within 2 months of DSE. These patients are the subject of this study. Study subjects ranged in age from 32 to 83 years (mean $60 \pm 12$ ), and 57 (41\%) were women. DSE was performed for evaluation of chest pain of uncertain etiology in 87 patients, for stratification after myocardial infarction in 15, for preoperative risk assessment in 14, for evaluation of interventional procedures in 14 and for miscellaneous indications in 11. Ninety-two patients were receiving antianginal therapy (either a $\beta$ blocker or calcium antagonist, or a combination) at the time of the study. Antianginal therapy was not discontinued before the study.

Coronary arteriography: All patients underwent cardiac catheterization (Judkins technique) with coronary arteriography within 2 months of DSE. Angiograms were reviewed by experienced angiographers using electronic calipers for measurement of vessel diameter and maximum narrowing, and for calculation of percent stenosis. Significant CAD was considered pres- 


\begin{tabular}{|c|c|c|c|}
\hline & Non- $\beta$-Blocked & $\beta$-Blocked & p Value* \\
\hline $\begin{array}{l}\text { Baseline systolic blood } \\
\text { pressure }(\mathrm{mm} \mathrm{Hg})\end{array}$ & $139 \pm 25$ & $122 \pm 19$ & $<0.001$ \\
\hline $\begin{array}{l}\text { Stress systolic blood } \\
\text { pressure (mm Hg) }\end{array}$ & $154 \pm 34$ & $142 \pm 27$ & 0.06 \\
\hline $\begin{array}{l}\text { Baseline heart rate } \\
\text { (beats } / \mathrm{min} \text { ) }\end{array}$ & $72 \pm 14$ & $63 \pm 11$ & $<0.001$ \\
\hline $\begin{array}{l}\text { Stress heart rate (beats/ } \\
\text { min) }\end{array}$ & $109 \pm 24$ & $85 \pm 20$ & $<0.0001$ \\
\hline
\end{tabular}

ent if a major epicardial coronary artery (right, left circumflex or left anterior descending, or a major branch of 1 of these distributions) had $\geq 50 \%$ diameter stenosis.

Dohutamine strese protocol: All patients were monitored continuously by a physician or registered nurse, or both, during the study. After a brief history and physical examination to exclude the presence of any contraindication to stress testing, an intravenous line was begun, and a 12-lead electrocardiogram was obtained. Dobutamine at a concentration of $1 \mathrm{mg} / \mathrm{ml}$ was prepared and infused, using an infusion pump. Patients were specifically queried for development of chest pain that was further categorized as typical angina, atypical chest pain or the pain for which the study had been recommended. Additional symptoms specifically queried included dyspnea, palpitations, flushing, tremulousness, nausea and lightheadedness. A complete 2-dimensional echocardiogram was recorded on videotape. For study

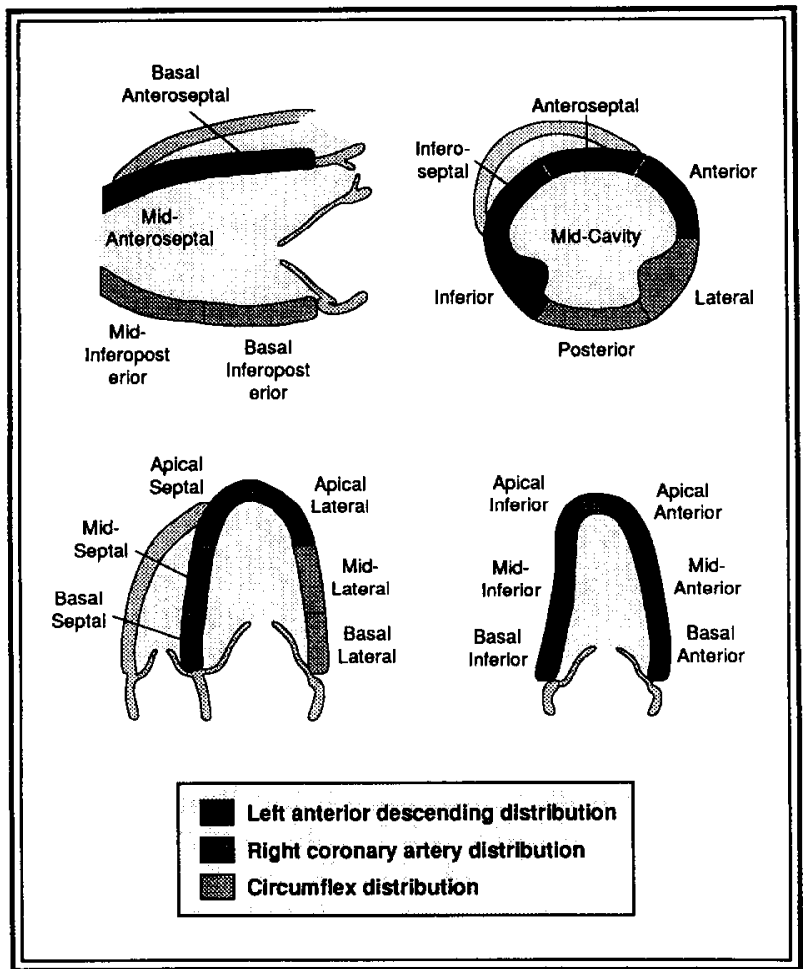

FGURE 1. Sixtoen-segment model depicting distribution of corenary artery territories. Depicted clockwise from top left are parasternd long- and short-axis, and 2- and 4-chamber viows. purposes, parasternal long- and short-axis, as well as apical 4- and 2-chamber views were recorded at 5, 10, 20 and $30 \mu \mathrm{g} / \mathrm{kg} / \mathrm{min}$ of dobutamine infusion on videotape. Images at baseline, 5 and $30 \mu \mathrm{g} / \mathrm{kg} / \mathrm{min}$ (or peak dose), and 5 minutes into recovery were transferred online in digital form for display as continuous loop images in a quadscreen format (Freeland Systems, Prism Imaging, Louisville, Colorado). Echocardiograms were recorded using commercially available 2-dimensional echocardiographic equipment with transducers ranging in frequency from 2.25 to $3.5 \mathrm{MHz}$. All studies were recorded on either an Advanced Technology Laboratory Ultra Mark IV or an Acuson XP-128/10. For those studies recorded on the Ultra Mark IV, the intrinsic digitizing capacity of the unit was used rather than the free-standing digitizing system. At the conclusion of the study, the quadscreen format images were transferred onto 3.5 inch disks for analysis. Furthermore, the videotape was analyzed for confirmation. All DSE was viewed by an experienced echocardiographer (WFA) with extensive experience in stress echocardiography. DSE was reviewed in a blinded fashion without knowledge of coronary arteriography, clinical history, electrocardiographic response or symptoms during infusion.

Echocardiographic wall motion was graded as normal, hypokinetic, akinetic or dyskinetic, and the presence or absence of aneurysm, thrombus or other structural heart disease was noted. Each echocardiogram was reviewed for wall motion, using the aforementioned scheme at each phase of stress. DSE was then categorized as either: (1) normal (all segments had normal motion at rest and developed hyperdynamic wall motion during dobutamine infusion), (2) ischemic (area of hypokinesis, akinesis or dyskinesis [not present at rest] developed during course of dobutamine infusion) or (3) fixed (resting abnormality was noted, which persisted in unchanged or worsened manner in 1 coronary segment). A previously described 16-segment model (Figure 1) was used for grading wall motion. ${ }^{10}$ Previous experience demonstrated that the ability to separate right coronary artery from left circumflex coronary stenosis using this scheme is limited, and for the purposes of analysis, the right and circumflex territories were combined as a posterior circulation for both angiographic and echocardiographic analyses. ${ }^{11}$

Electrocardiographic analysis: All patients had a 12-lead electrocardiogram recorded at rest and at each stage of dobutamine infusion. Electrocardiograms were evaluated by an experienced electrocardiographer who was blinded to the results of the echocardiogram. The resting electrocardiogram was evaluated for rhythm and evidence of prior myocardial infarction. Electrocardiographic changes diagnostic of myocardial ischemia were defined as $\geq 1 \mathrm{~mm}$ of horizontal or downsloping STsegment depression developing from a normal baseline, or $\geq 2 \mathrm{~mm}$ of additional ST-segment depression occurring in a lead with an abnormal configuration at rest.

\section{RESULTS}

Symptomatic response: DSE was well-tolerated in most patients. The study was not prematurely terminated for a side effect in any patient. Angina developed in 
17 patients (12\%), was not severe, and needed therapy with sublingual nitroglycerin in only 2 . Four patients developed symptomatic tachypnea or dyspnea, or both; all had previously documented chronic obstructive lung disease, and none needed inhaled bronchodilators. Other side effects were minor and self-limited, including anxiety, nausea without vomiting, tremulousness and skin tingling.

Hemodynamic and electrocardiographic results: Table I outlines the hemodynamic response to dobutamine infusion in the study population, divided into those patients receiving $\beta$-blocker therapy and those not on therapy. The hemodynamic and heart rate responses seen were modest compared with those expected during exercise testing. Hypotension (>20 $\mathrm{mm} \mathrm{Hg}$ decrease in blood pressure during protocol) occurred in $20 \%$ (568) of all patients studied and in $29 \%$ of those evaluated for this analysis. The magnitude of hypotension ranged from 20 to $90 \mathrm{~mm} \mathrm{Hg}$, occurred at a mean dose of $25 \pm$ $4 \mu \mathrm{g} / \mathrm{kg} / \mathrm{min}$ and was not associated with chest pain or shortness of breath.

Only 19 of 109 patients (17\%) with documented CAD had a typical ischemic response on the 12-lead electrocardiogram. New onset or increasing frequency of ventricular premature complexes was seen in $39 \mathrm{pa}$ tients (28\%); all were asymptomatic and returned to baseline frequency within 5 minutes of stopping dobutamine infusion. Five patients had supraventricular tachycardias, including 3 with atrial fibrillation or flutter; all except 1 ceased within 10 minutes of stopping dobutamine infusion.

Angiographic results: Significant $(\geq 50 \%$ diameter stenosis) CAD was present in 109 patients (77\%). This included 62 patients with 1-vessel, 21 with 2-vessel and 26 with 3-vessel CAD. Only 2 patients had left main coronary artery stenosis $\geq 50 \%$, and both had narrowing
FIGURE 2. Baceline and peak dobutamine Infudons in pationt with normal well motion at reat, and normal hypentymanic response to dobutamine. During systole (SYS), al segments thicken nonmaly and oxhibit normel luwerd motion. DAS = cadole.
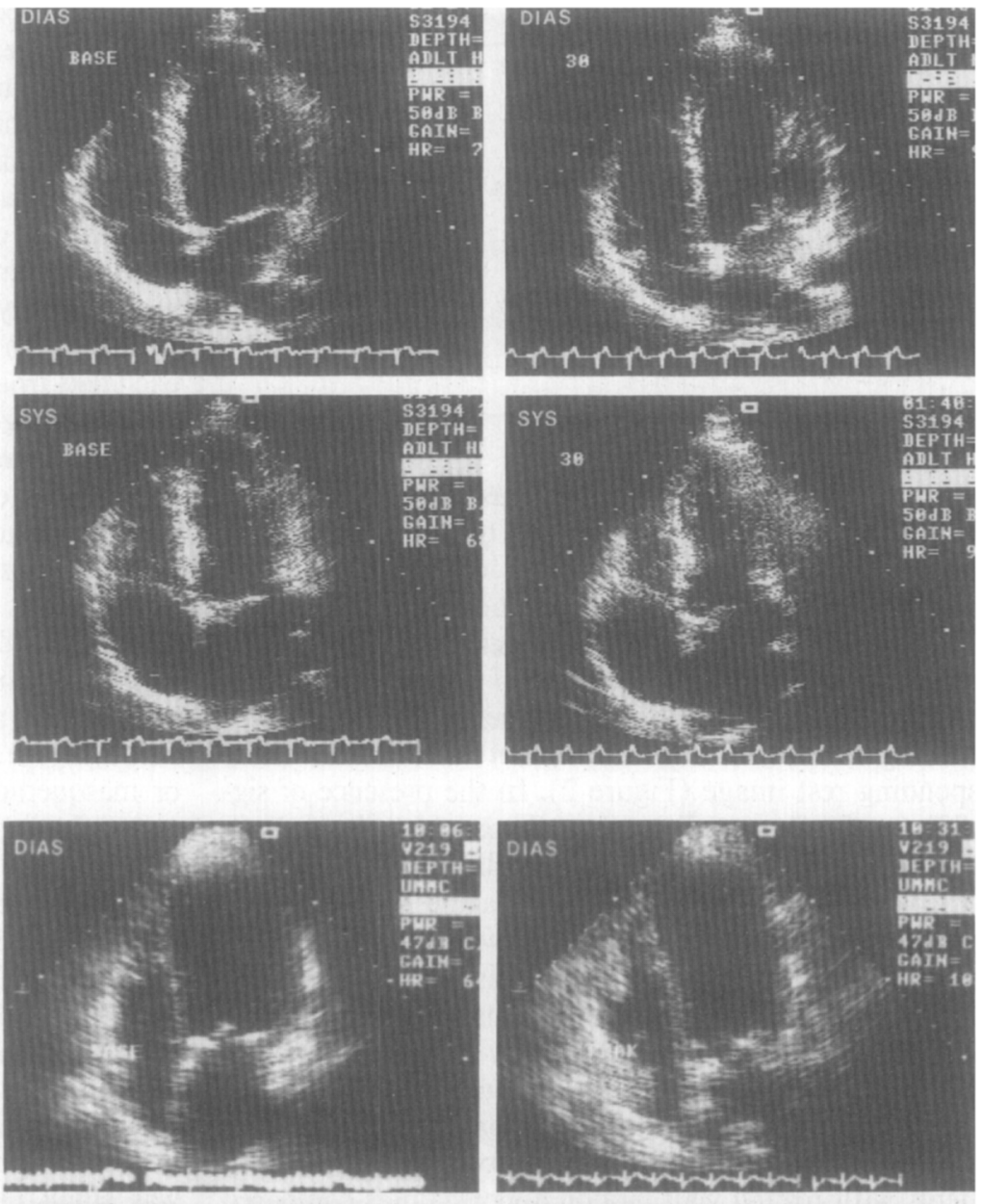

Givelon: in pationt with peak dobutanin oase in mid-teft anterlor deccending coronery artery. Al segments contract normalhy at baseline. At pack doce, distal septum and spex become dyskinetic (arrows). Abbreviations as in Flgure 2.
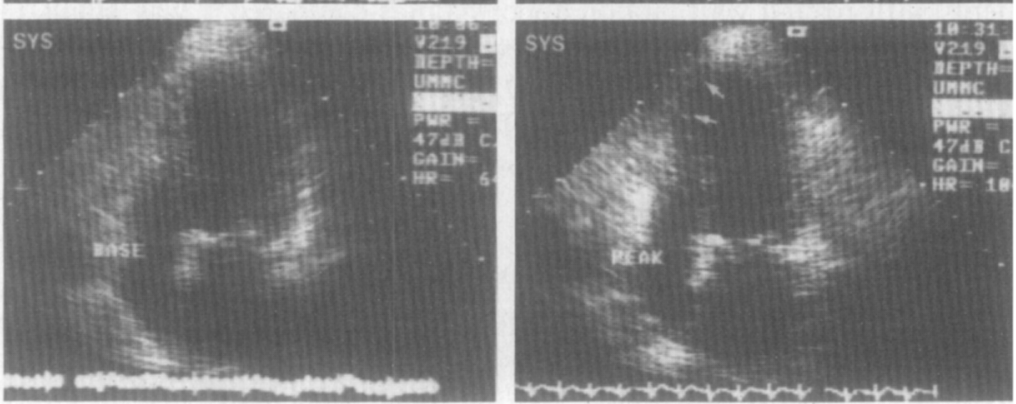


\begin{tabular}{|c|c|c|c|}
\hline & Sensitivity & Specificity & Accuracy \\
\hline $\begin{array}{l}\text { All patients under- } \\
\text { going cardiac } \\
\text { catheterization }\end{array}$ & $96 \%(105 / 109)$ & $66 \%(21 / 32)$ & $89 \%(126 / 141)$ \\
\hline 1-vessel disease & $95 \%$ & & \\
\hline Multivessel disease & $97 \%$ & & \\
\hline $\begin{array}{l}\text { Patients with nor- } \\
\text { mal resting wall } \\
\text { motion }\end{array}$ & $87 \%(26 / 30)$ & $91 \%(21 / 23)$ & $89 \%(47 / 53)$ \\
\hline Anterior circulation & $91 \%(59 / 65)$ & $79 \%(59 / 75)$ & $84 \%(118 / 141)$ \\
\hline Posterior circulation & $94 \%(73 / 78)$ & $71 \%(45 / 63)$ & $84 \%(118 / 141)$ \\
\hline
\end{tabular}

\begin{tabular}{|c|c|c|c|}
\hline & Sensitivity & Specificity & Accuracy \\
\hline $\begin{array}{l}\text { All patients receiving } \beta \\
\text { blockers or calcium } \\
\text { antagonists, or both } \\
(n=92)\end{array}$ & $96 \%(73 / 76)$ & $63 \%(11 / 16)$ & $91 \%(84 / 92)$ \\
\hline $\begin{array}{l}\text { All patients with NRWM } \\
\quad(n=43)\end{array}$ & $92 \%$ & $88 \%$ & $91 \%$ \\
\hline $\begin{array}{l}\text { NRWM: anterior circu- } \\
\text { lation }\end{array}$ & $82 \%$ & $97 \%$ & $93 \%$ \\
\hline $\begin{array}{l}\text { NRWM: posterior cir- } \\
\text { culation }\end{array}$ & $94 \%$ & $84 \%$ & $91 \%$ \\
\hline
\end{tabular}

in more distal coronary territories as well. The presence of $\mathrm{CAD}$ was known in 41 patients on the basis of prior myocardial infarction or previous catheterization and was previously undocumented in the remainder.

Dobutamine stress echocardiography: The normal response to dobutamine consists of hyperdynamic motion at peak dose, with all segments thickening and moving inward to a greater extent than in the corresponding rest image (Figure 2). In the presence of significant CAD, a wall motion abnormality develops in the region of the myocardium supplied by the critically stenosed vessel. Figure 3 depicts dyskinesis of the distal septum and apex in a 67-year-old woman with an $80 \%$ mid-left anterior descending stenosis. DSE was consistent with CAD in 105 of 109 patients with angiographic CAD. The sensitivity and specificity for the detection of CAD in the entire patient population, as well as those in the anterior and posterior circulations, are presented in Table II. Table II also shows the accuracy in those patients with resting wall motion abnormalities compared with that in those in whom only new ischemic changes were used in the diagnosis of CAD, and in those with single and multivessel CAD. Table III presents the sensitivity, specificity and accuracy for patients receiving active antianginal therapy.

False negative studies occurred in only 4 patients. The maximum heart rate during DSE was $<85$ beats/ $\min$ in 2 of these patients. A third patient had a supraventricular tachycardia during peak dobutamine infusion, increasing the complexity of wall motion analysis.
One patient had a technically poor study. False positive DSE was seen in 11 patients. Clinically documented cardiomyopathy or other forms of organic heart disease were present in 9 of these patients. One patient had critical aortic stenosis with left ventricular hypertrophy. The diagnosis of CAD in 7 of these 11 patients was based on the presence of wall motion abnormalities detected at rest (4 worsened during dobutamine infusion).

\section{Discussion}

Relative accuracy of dobutamine stress echocardiography: In this study, the accuracy, sensitivity, specificity and predictive value of DSE was equivalent to that of exercise echocardiography and competing nuclear medicine techniques. The sensitivity for detecting patients with single and multivessel CAD (95 and 97\%, respectively) was higher than previously reported values for the competing technologies ${ }^{1}$; however, the specificity in this patient population was only $66 \%$. Most false positive studies occurred in patients with wall motion abnormalities at rest. The current study was a blinded evaluation of wall motion abnormalitics in unsclected paticnts presenting for DSE. Patients were not excluded because of nonischemic heart disease, and all wall motion abnormalities were assumed to represent $\mathrm{CAD}$. This results in an obvious catheterization referral bias, because patients with an abnormal test are more likely to undergo arteriography than those with a normal result. When patients with an abnormal baseline echocardiogram are excluded from analysis, specificity increases to $91 \%$.

Advantages and limitations of dobutamine stress echocardiography! DSE has several advantages compared with other imaging techniques. As with other forms of echocardiographic stress testing, its versatility provides substantially more baseline data than do competing forms of cardiovascular stress testing. The baseline echocardiogram evaluates all 4 cardiac chambers and all 4 cardiac valves, and provides clues as to the presence or absence of virtually any form of organic heart disease. Virtually any cardiac disease that mimics or masquerades as CAD is accurately and quickly detected during the course of the stress echocardiogram.

Dobutamine (as used in this stress protocol) was very well-tolerated and was not associated with significant side effects. One of the major concerns regarding dobutamine infusion in patients with a high likelihood of CAD is that uncontrollable angina, myocardial infarction or significant arrhythmias may be precipitated. The benign side effect profile of DSE (as used in this protocol) would appear to diminish those concerns. The number of paticnts who devcloped angina during DSE was small $(12 \%)$, and when it occurred, angina was mild, needing sublingual nitroglycerin in only 2 patients. The incidence of induced angina is low compared with that in standard exercise testing. No patient had a prolonged episode of ischemia or myocardial infarction. Malignant arrhythmias (which have been a concern in the use of sympathomimetic agents) were not seen in this study.

An additional advantage of DSE is that it allows evaluation of left ventricular function and regional wall motion at multiple, incremental levels of left ventricular 
stress. Because the patient remains in the supine position throughout the study, body position can be optimized for high-quality echocardiographic images obtained at each incremental level of stress. This is not feasible using post-treadmill exercise imaging, where chest wall and respiratory motion sometimes makes obtaining consistently high-quality images difficult. Thus, the diagnostic yield may be higher than with post-treadmill or bicycle stress echocardiography. Furthermore, by analysis of the precise level of stress at which an ischemic wall motion abnormality develops, prognostic information may be available. ${ }^{12}$

The major limitation of DSE is that it relies on the induction of ischemia through increases in myocardial oxygen consumption to produce an abnormal result. This could theoretically lead to prolonged ischemia or infarction, or both. From this and previous studies, it appears that the magnitude of the induced ischemia is not sufficient to cause an escalating pattern of angina or myocardial infarction. An additional limitation of our study is that it represents a subjective evaluation of wall motion, rather than a detailed quantitative evaluation of wall motion abnormalities. The lack of quantitative data may hinder assessment of the precise onset of wall motion abnormalities. However, subjective interpretations are obtained in most clinical laboratories, and (when performed by experienced echocardiographers in a blinded fashion) provide accurate results regarding the presence and location of CAD. Precise quantitation of the magnitude of the ischemic burden may be necessary for prognostic purposes or for evaluating the results of an intervention. For diagnostic purposes, a simple qualitative assessment of wall motion (as in this study) suffices, and avoids the complexity, added cost and inconvenience of quantitative wall thickening or endocardial motion schemes. Furthermore, there is no consensus regarding the specific quantitative technique to use.

Comparison with other studies: DSE was first reported as a diagnostic technique by Berthe et $\mathrm{al}^{6}$ in 1986. They evaluated 30 patients 5 to 14 days after myocardial infarction to assess the accuracy of DSE for detection of multivessel CAD. Our results for the specific diagnosis of multivessel disease in patients with resting abnormalities (many of whom can be presumed to have had myocardial infarction) are virtually identical to theirs. Sawada et $\mathrm{a}^{8}$ also demonstrated the diagnostic use of DSE. They reported a sensitivity and specificity for detecting patients with CAD (92 and 79\%, respectively) that was similar to those reported here. The results reported here are fully in concordance with those of these other studies and suggest that DSE may have a valuable role in the evaluation of patients with known or suspected CAD.

\section{REFERENCES}

1. Kotler TS, Diamond GA. Exercise thallium-201 scintigraphy in the diagnosis and prognosis of coronary artery disease. Ann Intern Med 1990;113:684-702. 2. Armstrong WF, O'Donnell J, Dillon JC, McHenry PL, Morris SN, Feigenbaum $\mathbf{H}$. Complementary value of two-dimensional exercise echocardiography to routine treadmill exercise testing. Ann Intern Med 1986;105:829-835.

3. Limacher MC, Quinones MA, Poliner LR, Nelson JG, Winters WL, Waggoner AD. Detection of coronary artery disease with exercise two-dimensional echocardiography: description of a clinically applicable method and comparison with radionuclide ventriculography. Circulation 1983;67:1211-1218.

4. Crouse LJ, Harbrecht JJ, Vacek JL, Rosamond TL, Kramer PH. Exercise echocardiography as a screening test for coronary artery disease and correlation with coronary arteriography. Am $J$ Cardiol 1991;67:1213-1218.

5. Picano E, Lattanzi F, Masini M, Distante A, L'Abbate A. Usefulness of the dipyridamole-exercise echocardiography test for diagnosis of coronary artery disease. Am J Cardiol 1988;62:67-70

6. Berthe C, Pierard LA, Hiernaux M, Trotteur G, Lempereur P, Carlier J, Kulbertus HE. Predicting the extent and location of coronary artery disease in acute myocardial infarction by echocardiography during dobutamine infusion. Am J Cardiol 1986;58:1167-1172.

7. Mannering D, Cripps T, Leech G, Mehta N, Valentine H, Gilmour S, Bennett ED. The dobutamine stress test as an alternative to exercise testing after acute myocardial infarction. Br Heart $J$ 1988;59:521-526.

8. Sawada SG, Segar DS, Ryan T, Brown SE, Dohan AM, Williams R, Fineberg NS, Armstrong WF, Feigenbaum H. Echocardiographic detection of coronary artery disease during dobutamine infusion. Circulation 1991;83:1605-1614

9. Marcovitz PA, Mathias W, Dick R, Nath C, Armstrong WF. Accuracy of dobutamine stress echocardiography for the diagnosis of coronary artery disease: correlation with quantitative coronary arteriography (abstr). J Am Soc Echo $1991 ; 4: 279$.

10. Presti CF, Gentile RG, Armstrong WF, Ryan T, Dillon JC, Feigenbaum H. Improvement in regional wall motion after percutaneous transluminal coronary angioplasty during acute myocardial infarction: utility of two-dimensional echocardiography. Am Heart $J$ 1988; 115:1149-1155.

11. Armstrong WF, O'Donnell J, Ryan T, Feigenbaum H. Effect of prior myocardial infarction and extent and location of coronary disease on accuracy of exercise echocardiography. $J$ Am Coll Cardiol 1987;10:531-538.

12. Williams RL, Sawada SG, Lalka SG, Segar DS, Ryan T, Sebo M, Feigenbaum $\mathbf{H}$. Prognostic value of dobutamine stress echocardiography before vascular surgery (abstr). J Am Soc Echocardiography 1991;4:279. 